\title{
Necrotizing granulomatous inflammation: what does it mean if your special stains are negative?
}

\author{
Marie-Christine Aubry \\ Department of Laboratory Medicine and Pathology, Mayo Clinic, Rochester, MN, USA
}

\begin{abstract}
Necrotizing granulomas are commonly encountered in surgically resected specimens. The majority will be proven infectious with special stains for microorganisms. These need to be distinguished from other granulomatous processes such as Wegener's granulomatosis (WG). Although there may be histological overlap between these different processes, the identification of a true necrotizing vasculitis is specific to WG in the context of necrotizing granulomas. Otherwise, the combination of histological features should lead to a specific diagnosis. Despite a thorough histological examination and assessments of special stains, a significant proportion of necrotizing granulomas will appear infectious with no obvious infectious etiology. There are only few clinically available ancillary tests that can be performed on paraffin-embedded tissue and include real-time PCR for tuberculous mycobacteria. Despite correlation with clinical, serological and other microbiological studies, some necrotizing granulomas remain unexplained. Patients with such granulomas appear not to require any additional treatment and do experience a favorable outcome.

Modern Pathology (2012) 25, S31-S38; doi:10.1038/modpathol.2011.155
\end{abstract}

Keywords: fungi; granuloma; mycobacteria; Wegener's granulomatosis

Despite advances in radiological imaging up to $20 \%$ of resected pulmonary nodules are benign, and granulomas account for the majority of these nodules. Although the majority of necrotizing granulomas are infectious in etiology, Ulbright and Katzenstein ${ }^{1}$ found that $\sim 25 \%$ of these remained unexplained. Necrotizing granulomas of unexplained etiology remain part of our practice and the presentation at the USCAP long course tried to address three relevant questions: (1) What is the significance of a necrotizing granuloma that looks infectious but in which no organism is identified? (2) What features are most helpful in separating infectious granulomatous disease from other lesions such as Wegener's granulomatosis (WG)? And (3) are there newer techniques besides special stains and cultures to identify infectious agents? This review will focus on these three questions.

Correspondence: Dr M-C Aubry, MD, Department of Laboratory Medicine and Pathology, Mayo Clinic, 200 First Street SW, Rochester, MN 55905, USA.

E-mail: aubry.mariechristine@mayo.edu

Received 4 July 2011; accepted 6 September 2011

\section{What is the significance of necrotizing granuloma that looks infectious but in which no organism is identified?}

To answer this question, one needs to first answer the following: what is the significance of finding necrotizing granulomas in a resected lung specimen? Ulbright and Katzenstein ${ }^{1}$ looked at 86 consecutive necrotizing granulomas presenting as single nodules. They found that $61(71 \%)$ were proven infectious, 3 (3\%) were diagnosed as WG (1) and hyalinizing granuloma (2), and 22 (26\%) remained unexplained after clinical, radiological and microbiological correlation. In our practice (data not published), about half of necrotizing granulomas with negative special stains for microorganisms are infectious in etiology, $13 \%$ have another specific diagnosis and the number of unexplained necrotizing granulomas remains high at $39 \%$ (series of 132 patients). This leads us to pose the second question.

\section{What features are most helpful in separating infectious granulomatous disease from other lesions such as WG?}

Indeed, when presented with necrotizing granulomatous inflammation, a broad differential diagnosis 
Table 1 Overlap of histological features between infectious granulomas, unexplained granulomas, infarcts and other specific diseases

\begin{tabular}{|c|c|c|c|}
\hline Figure & $\begin{array}{l}\text { Infectious } \\
\text { granulomas } \\
\text { (\% cases) }\end{array}$ & $\begin{array}{c}\text { Unexplained } \\
\text { (\% cases) }\end{array}$ & $\begin{array}{l}d \text { Infarcts } \\
\text { (\% cases) }\end{array}$ \\
\hline Geographic necrosis & $23-30$ & $18-31$ & 35 \\
\hline 'Dirty' necrosis & 8 & $11-36$ & 52 \\
\hline Coagulative necrosis & 13 & 14 & 100 \\
\hline $\begin{array}{l}\text { Vascular inflammation } \\
\text { (including granulomas) }\end{array}$ & $36-59$ & $33-41$ & 56 \\
\hline Necrotizing vasculitis & 0 & 0 & 0 \\
\hline Thromboembolus & 0 & 0 & 100 \\
\hline $\begin{array}{l}\text { Lymphangitic distribution } \\
\text { of granulomas }\end{array}$ & 8 & 11 & NA \\
\hline
\end{tabular}

Abbreviation: NA, not applicable.

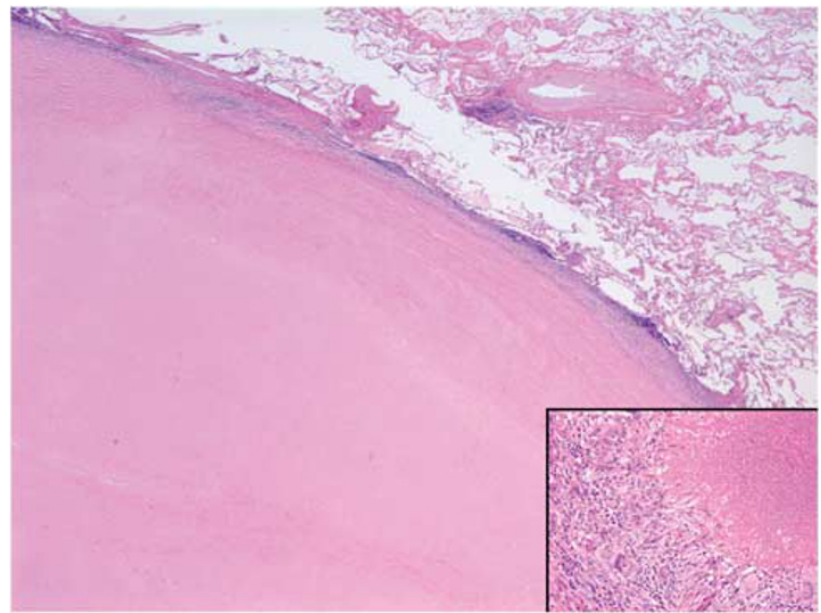

Figure 1 Low-power magnification of an infectious granuloma showing eosinophilic necrosis with regular rounded contour. The inset illustrates the rim of epithelioid histiocytes with multinucleated giant cells. $(\mathrm{H} \& \mathrm{E}, \times 40$; inset, $\times 400)$.

can be entertained by pathologists, most commonly infection, WG, aspiration pneumonia and less commonly rheumatoid nodule, necrotizing sarcoid granulomatosis (NSG), infarct and lymphomatoid granulomatosis, the latter being the topic of another review article in this issue. There are features that distinguish infectious granulomas from these other entities. However, overlapping features may cause diagnostic challenge and it is the combination of multiple histological findings that typically leads to the final diagnosis ${ }^{2}$ (Table 1). Infectious necrotizing granuloma usually has regular contours with mostly eosinophilic necrosis, surrounded by a rim of epithelioid histiocytes, often with multinucleated giant cells ${ }^{2}$ (Figure 1). However, in some cases, the contour of the necrosis may be more irregular (Figure 2a). Furthermore, the necrosis may take on different morphological aspect. It may be suppurative and rich in neutrophils, which may become necrotic and necrosis appear 'dirty' as seen in WG (Figure 2a) or it may be coagulative and mimics the
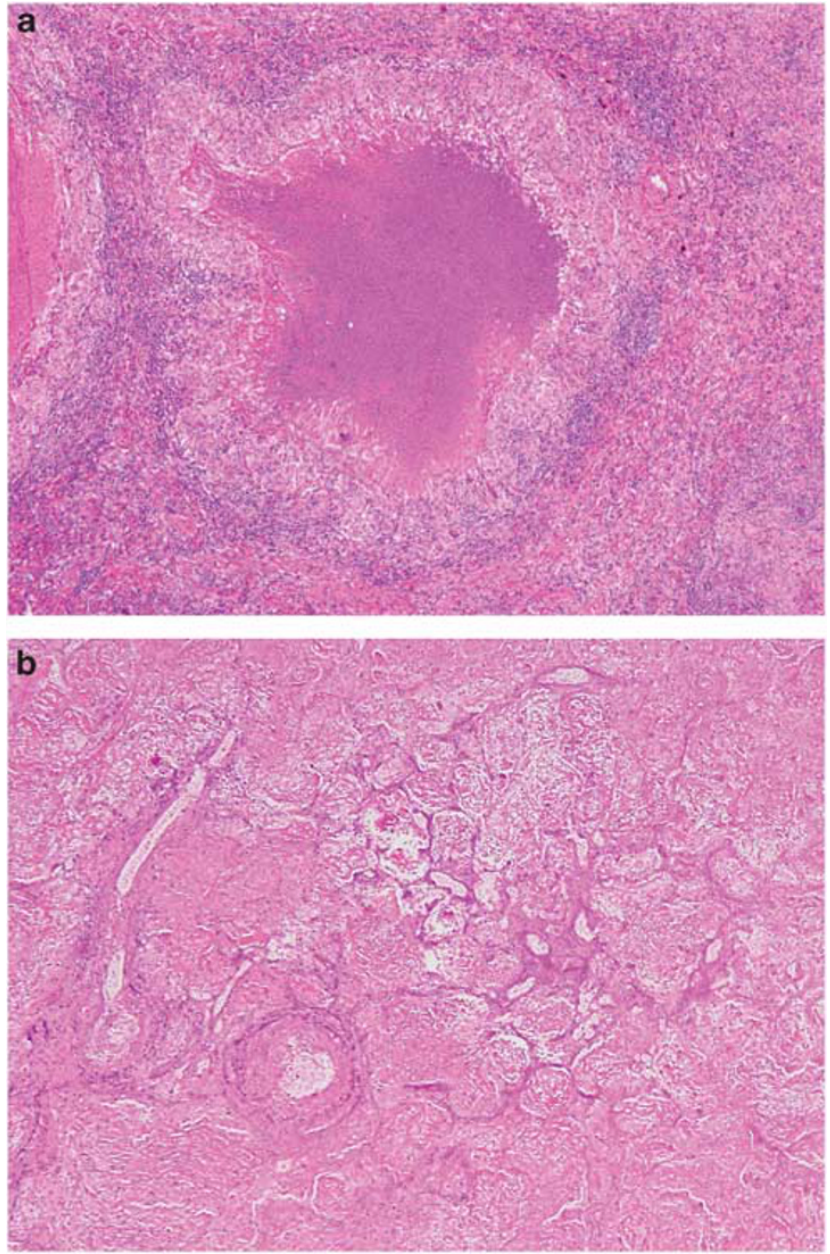

Figure 2 (a) At low power, this infectious granuloma displays more irregular contoured necrosis, which focally appears 'dirty' due to neutrophilic necrosis $(\mathrm{H} \& \mathrm{E}, \times 100)$. (b) The photomicrograph illustrates the center of an infectious granuloma with coagulative type of necrosis $(\mathrm{H} \& \mathrm{E}, \times 200)$

necrosis seen in arterial infarcts (Figure 2b). Vascular inflammation ('vasculitis') can be seen in infectious granulomas, usually chronic inflammatory cells with or without granulomas (Figure 3). However, a true necrotizing vasculitis characterized by fibrinoid necrosis of the media associated with necrotic neutrophils is not seen and is a specific feature of a true vasculitis such as WG. In contrast, WG is described by geographic necrosis, ie necrosis with irregular contours, and necrosis that is 'dirty' due to the intense necrosis of neutrophils with nuclear debris ${ }^{3}$ (Figure 4a). The necrosis is surrounded by a rim of palisading histiocytes and giant cells are common, often scattered, with hyperchromatic nuclei (Figure 4b). Furthermore, true necrotizing vasculitis is a distinctive feature (Figure 4c). Vessels located within the center of necrotic areas will exhibit true necrosis of their wall, however, these findings are seen in any necrotic process and are not diagnostic of a necrotizing vasculitis. In order to make this diagnosis, vessels more 

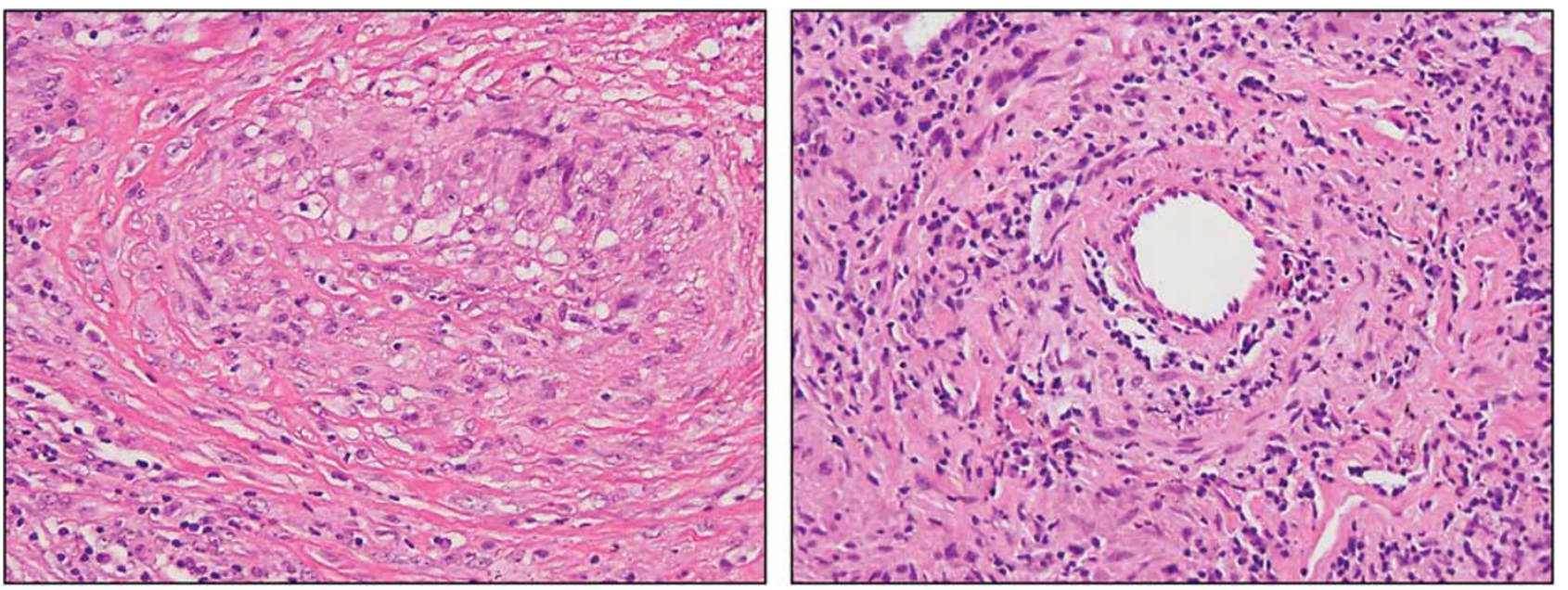

Figure 3 Photomicrographs illustrating vascular inflammation in infectious necrotizing granulomas. The walls of the vessels are thickened by chronic inflammatory cells including granulomatous inflammation. No fibrinoid necrosis of the media or necrotic neutrophils, features of a true necrotizing vasculitis, is identified $(\mathrm{H} \& \mathrm{E}, \times 400)$.
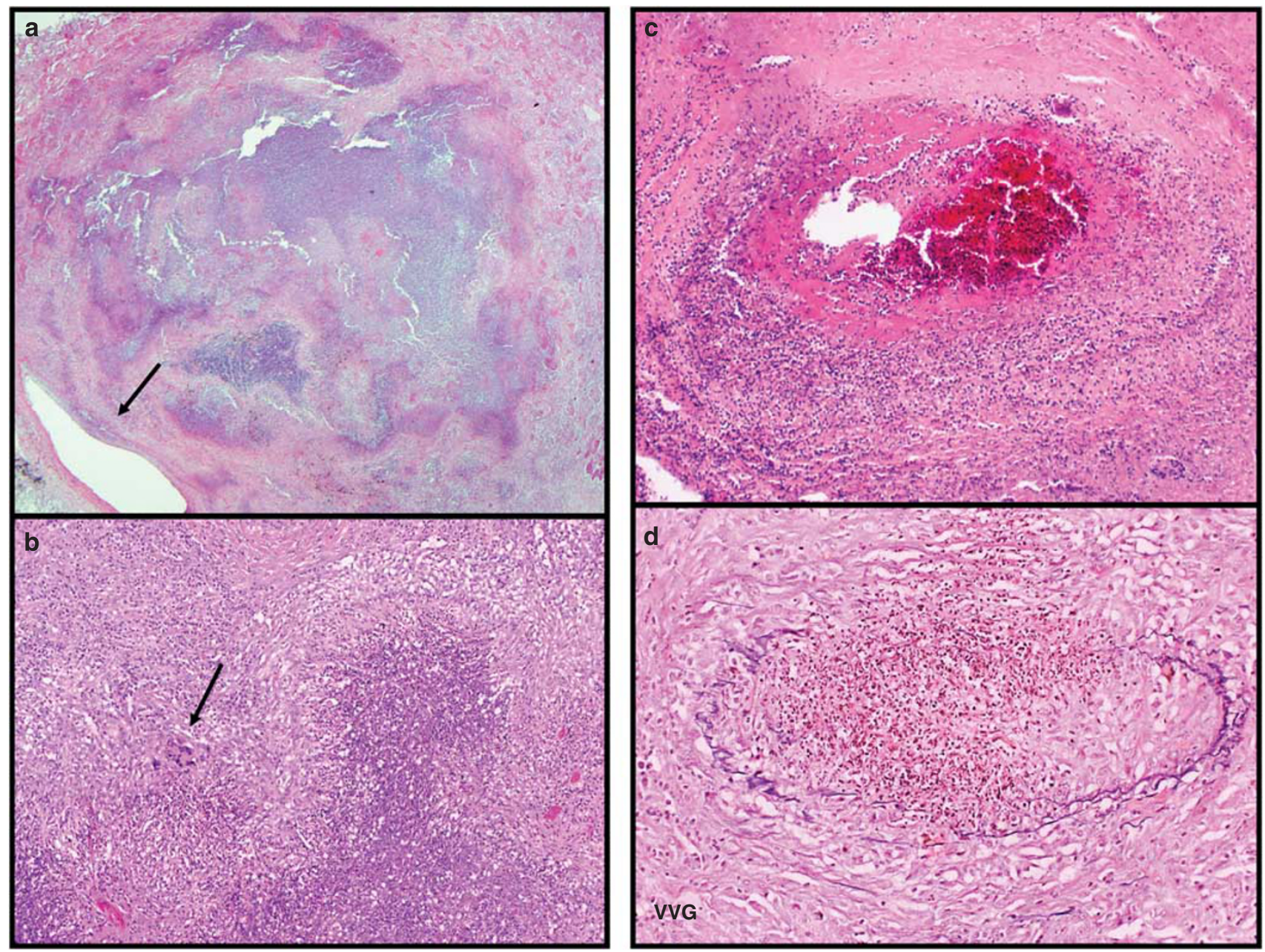

Figure 4 (a) Wegener's granulomatosis is characterized by 'dirty' basophilic necrosis with irregular geographic necrosis. This prompts the search for necrotizing vasculitis in vessels more peripheral to the necrosis (arrow; H\&E, $\times 20$ ). (b) The necrosis is rimmed by palisading histiocytes and scattered multinucleated giant cells are easily seen even at low power because of the hyperchromatic nuclei (arrow; H\&E, $\times 100$ ). (c) Vessels with true necrotizing vasculitis display fibrinoid necrosis of the media with admixed necrotic neutrophils. (d) An elastic stain helps in assessing transmural necrosis and inflammation of portion of the vascular wall (H\&E, $\times 100$ and VVG, $\times 200)$. 

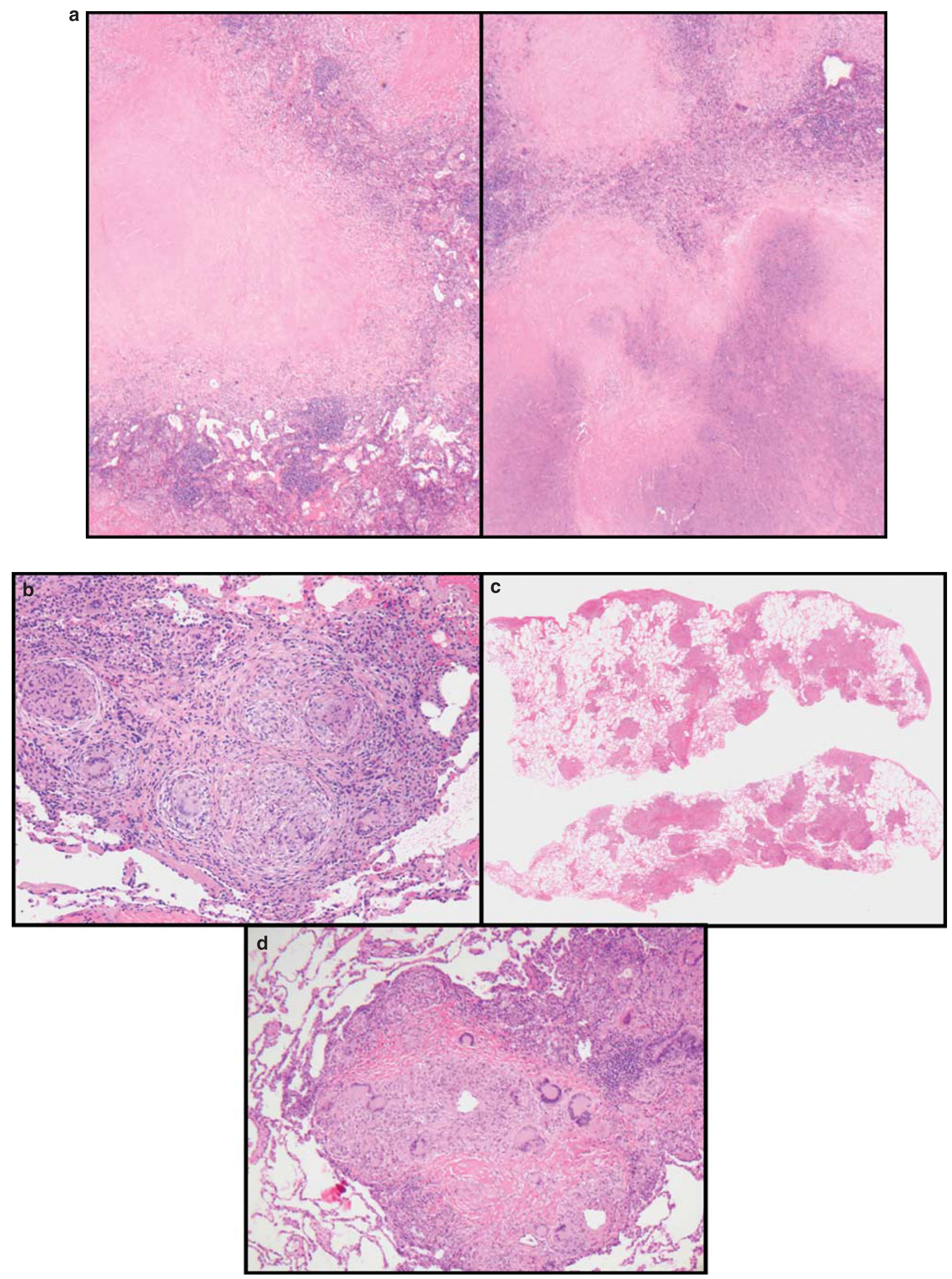
peripheral to the necrosis need to be assessed and an elastic stain such as Verhoeff von Gieson may be helpful. Features that can also assist in distinguishing infectious necrotizing granulomas from WG are the presence of non-necrotizing granulomas and lymph node involvement by granulomatous inflammation seen in infection and typically not in WG. NSG is a rare entity, considered as a variant of sarcoidosis $^{4}$ (Figure 5). It shares overlapping features with infectious granulomas and WG. Indeed, parenchymal necrosis is typical of NSG and the morphological appearance is variable, usually described as eosinophilic but may also be basophilic with nuclear debris. However, this necrosis occurs in a background of non-necrotizing granulomas as seen in sarcoidosis with granulomas displaying a lymphangitic distribution and being well-formed, composed predominantly of epithelioid histiocytes and giant cells with few intervening lymphocytes or other inflammatory cells. In NSG, these granulomas tend to be confluent and prominently involve vessels but without resulting in a true necrotizing vasculitis. As infectious necrotizing granulomas may exhibit coagulative type necrosis, another important differential diagnosis is arterial infarct (Figure 6), particularly in a small biopsy specimen where sampling may be an issue. ${ }^{5}$ Furthermore, infarcts as they organize may be surrounded by a rim of fibroblasts and inflammatory cells which can mimic granulomatous inflammation and described

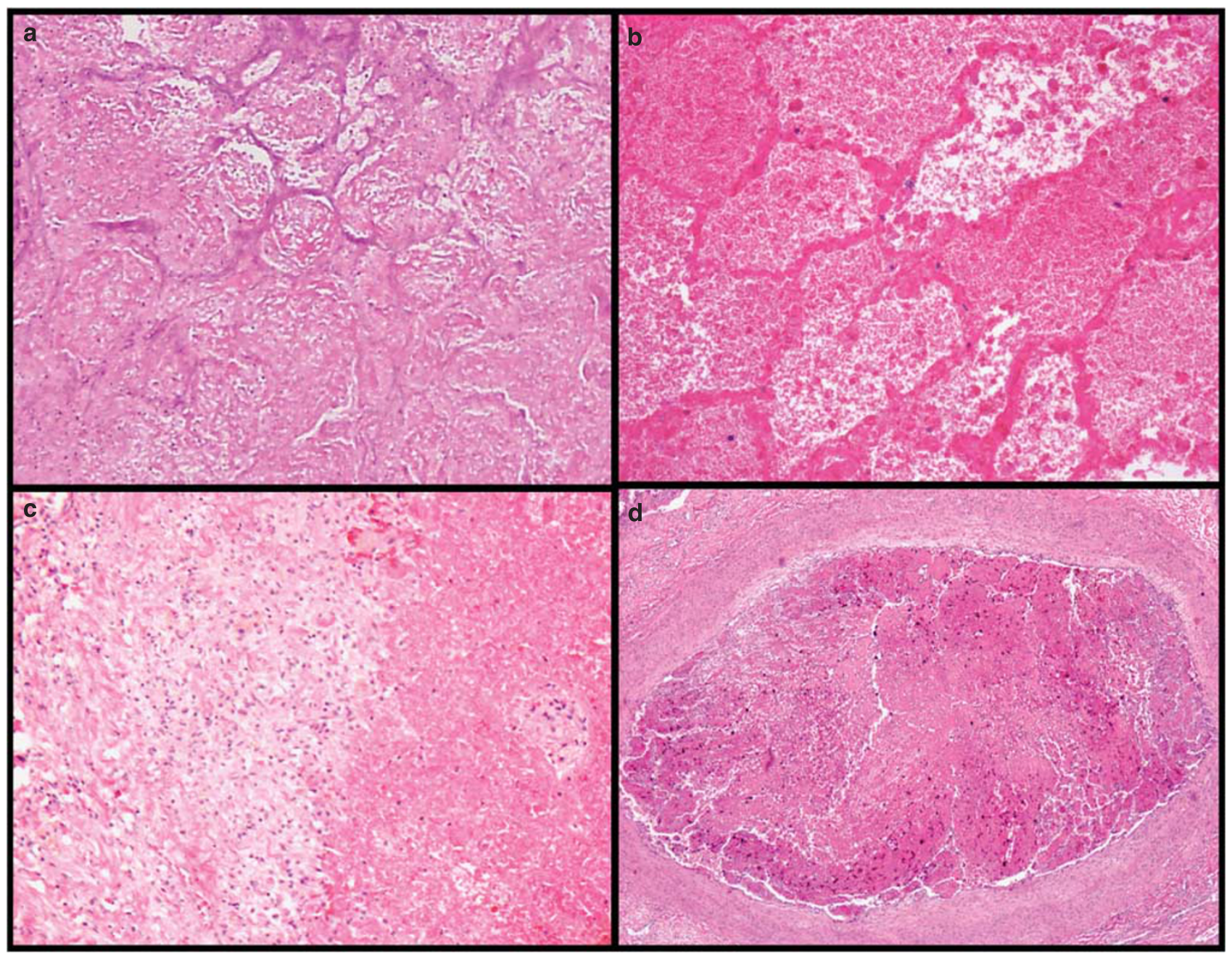

Figure 6 (a) Coagulative necrosis seen in necrotizing granuloma (H\&E, $\times 200)$. (b) In comparison, coagulative necrosis seen in an infarct $(\mathrm{H} \& \mathrm{E}, \times 200)$. (c) The periphery of an organizing infarct showing fibroblasts and chronic inflammatory cells that may mimic the rim of histiocytes seen in granulomas $(\mathrm{H} \& \mathrm{E}, \times 200)$. (d) In surgically resected specimens of infarcts, a thrombosed artery is typically seen $(\mathrm{H} \& \mathrm{E}, \times 40)$.

\footnotetext{
Figure 5 (a) Parenchymal necrosis of necrotizing sarcoid granulomatosis (NSG) is variable, usually eosinophilic but can be irregular and basophilic, mimicking Wegener's granulomatosis (H\&E, $\times 40)$. (b) This parenchymal necrosis is associated with a background of nonnecrotizing sarcoid type granulomas in a lymphangitic distribution $(H \& E, \times 200)$. (c) The non-necrotizing granulomatous inflammation displays a characteristic lymphangitic distribution $(\mathrm{H} \& \mathrm{E}, \times 1)$. (d) Prominent vascular involvement by the non-necrotizing granulomas is typical of NSG $(\mathrm{H} \& \mathrm{E}, \times 100)$.
} 
as 'pseudo granulomatous'. In a study of wedge resection specimens, arterial thrombosis was seen in all cases of infarcts, a feature that would distinguish infarct from infectious necrotizing granuloma. ${ }^{5}$

In summary, there is often no single histological feature that distinguishes infectious necrotizing granulomas from other specific disorder, with the exception of true necrotizing vasculitis that, in the context of necrotizing granulomas, would support the diagnosis of WG. It is the combination of multiple features, which can lead the pathologist towards a specific diagnosis. However, once all specific diagnosis have been eliminated, there will remain a significant number of necrotizing granulomas that appear infectious but with negative special stains for microorganisms. This leads us to pose the next question:

\section{Are there newer techniques besides special stains and cultures to identify infectious agents?}

Before answering this question, there are a few things to consider when assessing special stains, typically Grocott Methamine Silver (GMS) and Zhiel-Nelson (ZN), for microorganisms and increasing the sensitivity of our diagnosis. For fungi, it is helpful to look at the necrosis on H\&E slides before looking at the stains. Many fungi such as Cryptococcus, Blastomyces, Coccidioides and Aspergillus can be seen on H\&E, preferentially in the area of necrosis rather than the surrounding viable area. ${ }^{6}$ When assessing special stains, necrosis should be present on the slides, as this is the area where organisms are likely to be. In a study of 17 cases, Goodwin et $\mathrm{al}^{7}$ increased the sensitivity of a positive silver stain for Histoplasma by 59\% simply by insuring that necrosis was present on the slides. Fungi, especially Histoplasma, may be few and necrotic with weak staining thus not easily visible (Figure 7). Detecting these rare fungi requires more than a cursory scanning at low power and confirmation often needs to be performed at high magnification. Re-examination of stains is also useful and can increase detection of Histoplasma in up to $90 \%$ of cases. ${ }^{1}$ Mycobacteria are also often few and difficult to find. Some authors have even recommended scanning with high-power oil immersion. ${ }^{8}$ However, in practice, scanning with a $\times 40$ objective should suffice in most cases. Alternative stains, such as auramine/auramine-rhodamine (AR) using fluorescence technique, have been proposed as some studies have suggested increase sensitivity of $A R$ compared with conventional ZN. ${ }^{6,9}$ Although certainly easier to identify rare organisms on AR stain (Figure 8), there are issues related to sampling and leveling that may affect the sensitivity of a stain in the detection of rare organisms. Performing stains on more than one block can also increase the sensitivity of identifying microorganisms (Figure 9). Based on

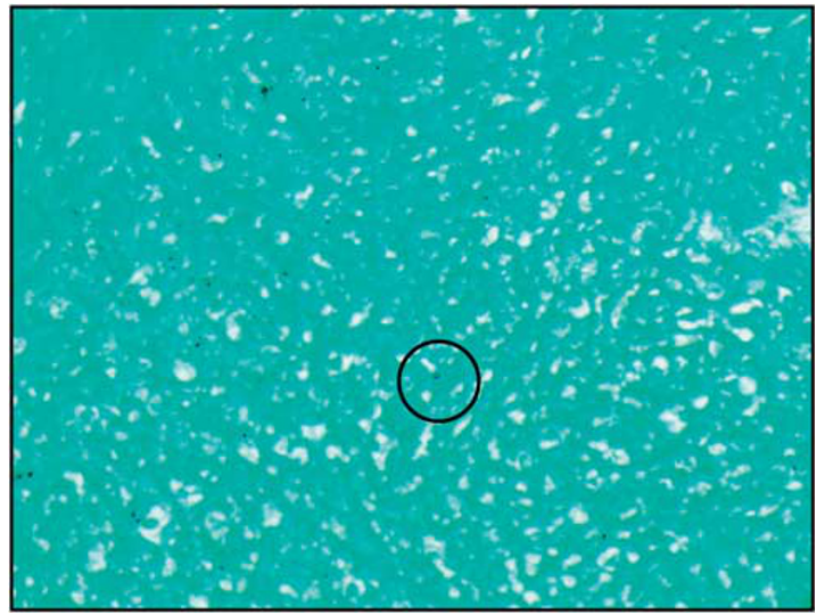

Figure 7 High-power photomicrograph showing a single yeast (in circle) consistent with Histoplasma in a large necrotizing granuloma (Grocott Methamine Silver, $\times 600$ ).

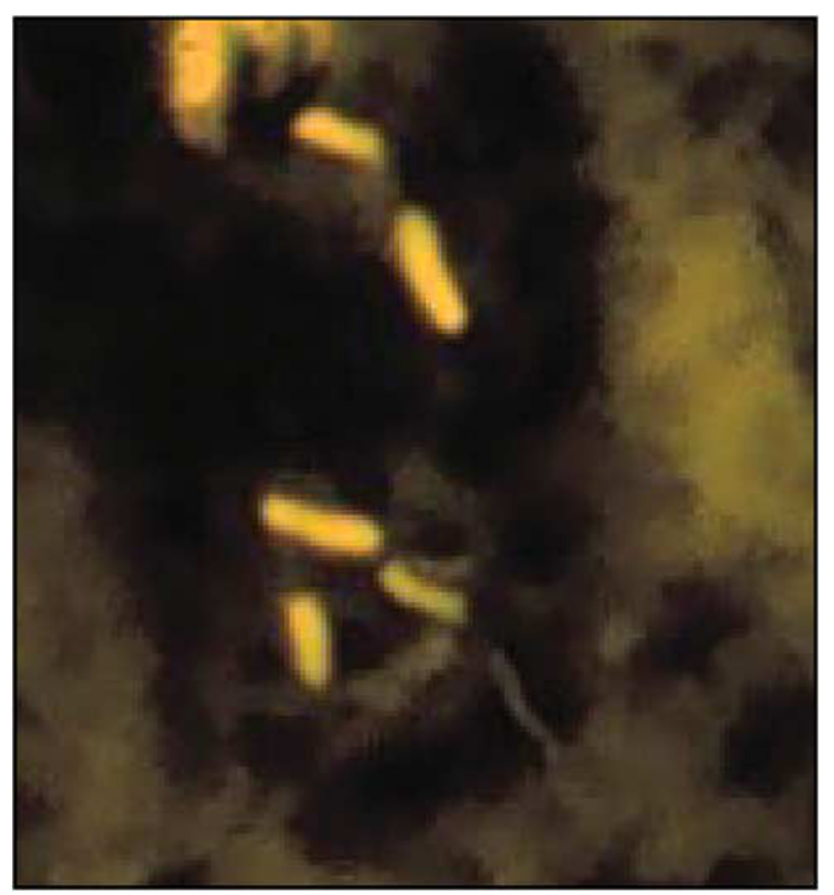

Figure 8 High-power photomicrograph showing mycobacteria on an auramine-rhodamine stain (AR, $\times 600)$.

their results, Ulbright and Katzenstein ${ }^{1}$ recommended doing stains on at least two blocks.

There are few clinically available ancillary studies that can be performed on paraffin-embedded tissue to complement special stains in the identification of organisms. Immunohistochemistry for detection of microorganisms is available but with several limitations, including cross-reactivity and possible decreased specificity and lack of available antibodies, more specifically for usual microorganisms resulting in necrotizing granulomatous inflammation. ${ }^{10}$ In-situ hybridization (ISH) can be useful in the speciation but not the detection of fungi 


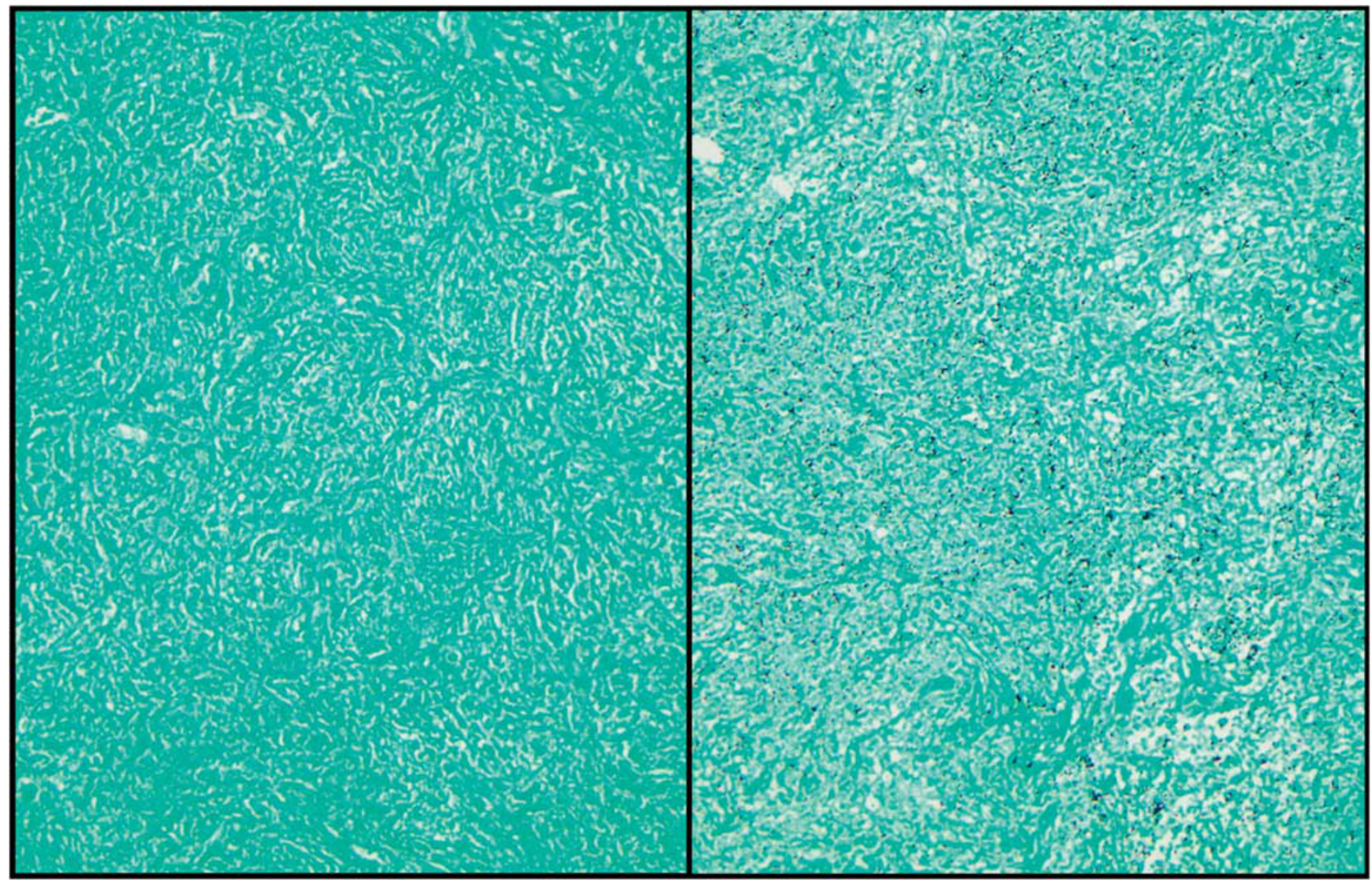

Figure 9 Intermediate-power photomicrograph of two different blocks stained with Grocott Methamine Silver (GMS). The first GMS is negative whereas the second shows abundant yeasts c/w Histoplasma (GMS, $\times 200$ ).

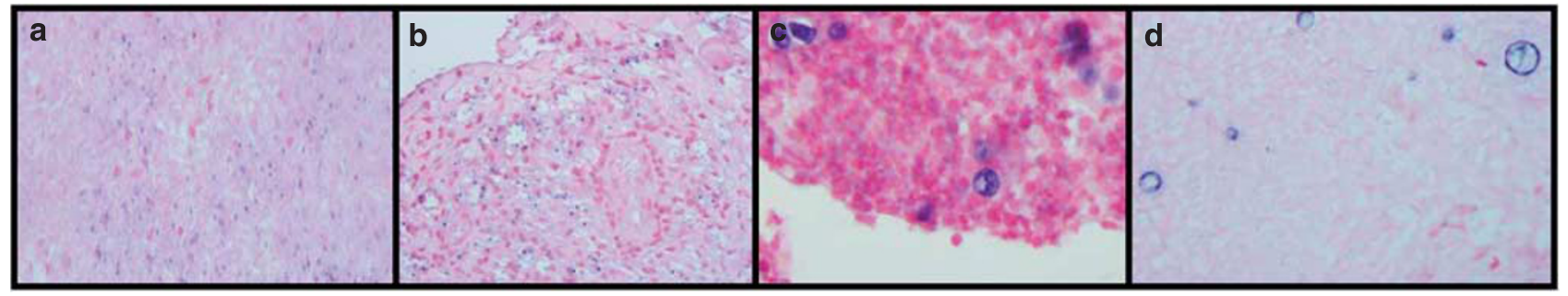

Figure 10 Photomicrographs of in-situ hybridization for various fungi: (a) Histoplasma, (b) Cryptococcus, (c) Blastomyces and (d) Coccidioidomyces.

(Figure 10). Indeed, ISH is useful only if the fungi are identified on H\&E or GMS but their subtyping poses a challenge. False negative results are an issue and sensitivity varies with the probes between 75-95\%. ${ }^{11}$ Real-time PCR for Histoplasma is available but requires fresh frozen tissue. ${ }^{12}$ For mycobacteria, detection of these small and few microorganisms on conventional $\mathrm{ZN}$ is a real issue and screening may be laborious. Searching for a method to facilitate screening, Dr Tadrous reported an automatic screening algorithm using digital image analysis. ${ }^{13}$ This methodology was very sensitive, capable of detecting a single bacillus in multiple images. This study requires further validation on a larger cohort of patients but seems promising. Real-time PCR for tuberculous mycobacteria on paraffin-embedded tissue is available with a high specificity of $99 \%$ but low sensitivity of $65 \%$ in contrast to other types of specimens such as cerebral spinal fluid, urine and bronchoalveolar lavage where sensitivity of $>90 \%$ is reported, with comparable specificity. ${ }^{14,15}$ So this test is useful if positive and confirms the presence of tuberculous mycobacteria but if negative, does not rule out the possibility of an infection. Furthermore, it does not detect non-tuberculous mycobacteria. Therefore, culture remains the gold standard for mycobacterial infection and in our experience was the only positive test in $>75 \%$ of cases, usually tissue culture. Furthermore, non-tuberculous infections is now more common than tuberculosis in Western countries and therefore would not be detected with this assay. ${ }^{16,17}$ 


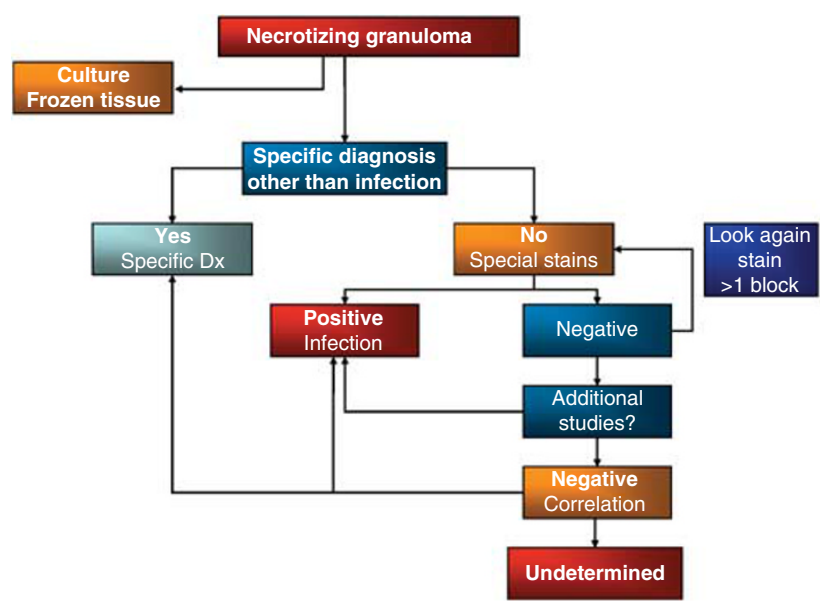

Figure 11 Algorithm for diagnostic approach to necrotizing granulomatous inflammation.

Despite a complete histological evaluation with clinical, microbiological and serological correlation, a significant proportion of necrotizing granulomas remain undetermined and this brings us full circle to the first question: What is the significance of necrotizing granuloma that looks infectious but in which no organism is identified?

Ulbright and Katzenstein ${ }^{1}$ suggested that these cases represent infectious granulomas in which microorganisms have been killed and/or removed by the inflammatory process. In a follow-up of 22 of their cases, no patients received treatment and all were alive and well. In our practice, we have experienced a similar outcome, with patients not receiving additional treatment, even in cases with microorganisms identified retrospectively on special stains, and being all alive and well. Rarely, new nodules were noted but even then, these patients were simply followed and did not experience any unfavorable outcome.

In summary (Figure 11), ideally, a surgically resected necrotizing granuloma is received fresh as to offer the opportunity to collect tissue for culture and also, if possible, snap-frozen tissue. When reviewing the histology of a necrotizing granuloma, a first step is to determine whether the granuloma appears infectious or it has other features leading to a specific diagnosis such as WG. If a specific diagnosis is ruled out, special stains for microorganisms are performed. Consider staining more than one block and insuring that necrosis is present on the stains. At first glance, if stains appear negative, it may be worth looking again and scanning at higher power. If despite this, stains remain negative and there is a high clinical suspicion for an infection, such as tuberculosis, real-time PCR can be performed. If the result is negative, correlating with cultures, serologies and other clinical tests may lead to a specific diagnosis including infection. At the end of this thorough algorithm, a certain number of necrotizing granulomas remained unexplained.
These patients do not appear to require any additional treatment and follow-up indicates a favorable outcome.

\section{Disclosure/conflict of interest}

This author declares no conflict of interest.

\section{References}

1 Ulbright TM, Katzenstein AA. Solitary necrotizing granulomas on the lung. Am J of Surg Pathol 1980;4: 13-28.

2 Mukhopadhyay S, Gal AA. Granulomatous lung disease: an approach to the differential diagnosis. Arch Pathol Lab Med 2010;134:667-690.

3 Yi ES, Colby TV. Wegener's granulomatosis. Semin Diag Pathol 2001;18:34-46.

4 Ma Y, Gal A, Koss MN. The pathology of pulmonary sarcoidosis: update. Semin Diag Pathol 2007;24:150-161.

5 Yousem SA. The surgical pathology of pulmonary infarcts: diagnostic confusion with granulomatous disease, vasculitis, and neoplasia. Mod Pathol 2009;22: 679-685.

6 Woodard BH, Rosenberg SI, Farham R, et al. Incidence and nature of primary granulomatous inflammation in surgically removed material. Am J Surg Pathol 1982;6: 119-129.

7 Goodwin Jr RA, Snell JD, Hubbard WW, et al. Early chronic pulmonary histoplasmosis. Am Rev Respir Dis 1966;93:47-61.

8 Nayak NC, Sabharwal BD, Bhatena D, et al. The pulmonary tuberculous lesion in North India: a study in medico-legal autopsies. I. incidence, nature, and evolution. Am Rev Respir Dis 1970;101:1-17.

9 Kommareddi S, Abramowsky CR, Swineheart GL, et al. Nontuberculous mycobacterial infections: comparison of the fluorescent auramine-O and Ziehl-Neelsen techniques in tissue diagnosis. Hum Pathol 1984;15: 1085-1089.

10 Eyzaguirre E, Haque AK. Application of immunohistochemistry to infections. Arch Pathol Lab Med 2008; 132:424-431.

11 Hayden RT, Qian X, Roberts GD, et al. In situ hybridization for the identification of yeast-like organisms in tissue section. Diag Mol Pathol 2001;10:15-23.

12 Kauffman CA. Histoplasmosis: a clinical and laboratory update. Clin Micro Reviews 2007;20:115-132.

13 Tadrous PJ. Computer-assisted screening of ZN-stained tissue for mycobacteria. Algorithm design and preliminary studies on 2,000 images. Am J Clin Pathol 2010;133:849-858.

14 Nyendak MR, Lewinsohn DA, Lewinsohn DM. New diagnostic methods for tuberculosis. Curr Opin Infect Dis 2009;22:174-182.

15 Buckwalter SP, Wengenack NL. Detection of mycobacteria in paraffin-embedded tissue using lightcycler PCR. American Society for Microbiology. 104th General Meeting. 23-27 May, 2004; New Orleans, LA.

16 Griffith DE. Nontuberculous mycobacterial lung disease. Curr Opin Infect Dis 2010;23:185-190.

17 Jarzembowski JA, Young MB. Nontuberculous mycobacterial infection. Arch Pathol Lab Med 2008;132: 1333-1341. 